\title{
Metastasis Progression Through the Interplay Between the Immune System and Epithelial- mesenchymal-transition in Circulating Breast Tumor Cells
}

\section{Samane Khoshbakht}

Laboratory of Systems Biology and Bioinformatics (LBB), Department of Bioinformatics, Kish International Campus, University of Tehran, Kish Island, Iran https://orcid.org/0000-0003-3253-7577 Sadegh Azimzadeh Jamalkandi ( $\sim$ azimzadeh@bmsu.ac.ir)

Chemical Injuries Research Center, Systems Biology and Poisonings Institute, Tehran, Iran https://orcid.org/0000-0003-3403-3700

\section{Ali Masudi-Nejad}

Laboratory of Systems Biology and Bioinformatics (LBB), Institute of Biochemistry and Biophysics, University of Tehran, Tehran, Iran

\section{Research article}

Keywords: breast cancer, single / cluster CTC, metastasis, co-expression, epithelial-mesenchymal transition, immune response

Posted Date: September 21st, 2020

DOl: https://doi.org/10.21203/rs.3.rs-74787/v1

License: (c) (i) This work is licensed under a Creative Commons Attribution 4.0 International License. Read Full License 


\section{Metastasis progression through the interplay between the}

\section{2immune system and Epithelial-Mesenchymal-Transition in}

\section{circulating breast tumor cells}

1. Laboratory of Systems Biology and Bioinformatics (LBB), Department of Bioinformatics, Kish International Campus, University of Tehran, Kish Island, Iran, samane.khoshbakht@ut.ac.ir

2. Chemical Injuries Research Center, Systems Biology and Poisonings Institute, Tehran, Iran, amasoudin@ut.ac.ir

3. Laboratory of Systems Biology and Bioinformatics (LBB), Institute of 


\section{Abstract}

\section{Background}

23 Circulating tumor cells (CTCs) are the critical initiators of distant metastasis formation. In

24 which, the reciprocal interplay among different metastatic pathways which promote survival 25 of CTCs, is not well introduced, using network approaches. CTC cells include single and 26 cluster cells, in which cluster cells revealed 23-50 fold more metastatic potentials.

27 Here, to investigate the unknown pathways of single/cluster CTCs, the co-expression network reconstructed, using WGCNA (Weighted Correlation Network Analysis) method. Having used the hierarchical clustering, we detected the Immune-response and EMT subnetworks. The metastatic potential of genes was assessed and validated through the support vector machine

31 (SVM), neural network, and decision tree methods on two external datasets. To identify the

32 active signaling pathways in CTCs, we reconstructed a casual network. The Log-Rank test and 33 Kaplan-Meier curve were applied to detect prognostic gene signatures for metastasis-free survival. Finally, a predictive model was developed for metastasis risk of patients, using VIFstepwise feature selection.

\section{Results}

37 Our results showed the crosstalk among EMT, the immune system, menstrual cycles, and the 38 stemness pathway in CTCs. In which, fluctuation of menstrual cycles is a new detected 39 pathway in breast cancer CTCs. The reciprocal association between immune responses and 40 EMT was identified in single/cluster CTCs. The SVM model indicated a high metastatic 41 potential of EMT subnetwork (accuracy, sensitivity, and specificity scores were 87\%). The 42 distant-metastasis-free-survival model was identified to predict patients' metastasis risks. (c43 index=0.8). Finally, novel metastatic biomarkers including PTCRA, F13A1, ICAM2, and 44 SNRPC were detected in breast cancer. 


\section{Conclusions}

46 In conclusion, the reciprocal interplay among critical pathways in CTCs enhances their 47 survival and metastatic potentials. Such findings may help to develop more precise predictive 48 metastatic-risk models or detect novel biomarkers.

\section{KEYWORDS}

50 breast cancer, single / cluster CTC, metastasis, co-expression, epithelial-mesenchymal transition, immune response

\section{Background}

53 Metastasis is the leading cause of death among women with breast cancer [1, 2]. Cancer progression and metastasis are of the critical and even controversial aspects of cancer studies [2]. There are two arguable metastasis models, including parallel progression and linear progression, which try to explain the dark side of the tumor developments [3]. In the linear model, the tumor initiates by genetic or epigenetic alternations, grows, spreads, and gains metastasis potentials to disseminate ectopic sites; Contrarily, in the parallel model, the metastasis ability initiates early-onset and evolves by circulating tumor cells (CTCs), parallelly $[3,4]$. CTCs, which negatively relate to the high rise of mortality rates in cancer, are rare disseminated tumor cells in the peripheral blood of patients [5]. Of note, they appear even in

62 the early stages and are prominent and leading components in metastasis $[2,6]$. Therefore, the

63 detection of CTCs in metastatic and non-metastatic breast cancer patients implies their leading

64 role in cancer progression [7]; Moreover, their physical characteristics as single CTC or CTC clusters play a crucial role in metastasis propensity [5]. They borrow the morphologic features

66 of their primary tumors and gain new features to survive in blood [8]. CTC clusters, which

67 consist of 2-50 cancer cells, can transit through the circulation of patients and increase the 68 potential of metastasis to 23- to 50-fold [5]. They overcome many hurdles to colonize distant 
organs including intravasation into circulation, evading immune bulwarks, extravasation to

70

71 distant sites, and eventually replacing the microenvironment of host tissue $[9,10]$.

Of note, the signaling pathways or intrinsic molecular characteristics of the single/cluster CTCs are not well recognized. Therefore, fully realize the CTCs' cellular features, using network approaches, will guide us to unknown metastasis concepts and more precise therapeutic decisions; In which, the reversal phenotypic of Epithelial-Mesenchymal-Transition (EMT) or immune system are two prominent components in cancer progression $[1,11,12]$. EMT mechanism, which helps cancer cells lose their cell adhesion and gain mesenchymal phenotype, accelerates metastasis through immunosuppression in primary tumors [12-14]. Accordingly, assessment of the role of EMT and immune responses in CTCs as well as intermediate pathways is essential in cancer biology.

In this study, we implemented the co-expression network reconstruction for CTCs isolated from advanced patients' blood, using WGCNA method. We extracted metastasis relevant subnetworks that enriched in the immune system and EMT pathways. The preservation of subnetworks was assessed in GSE51827. The metastasis-free survival analysis and KaplanMeier curve of genes were implemented in GSE7390 (external data). Concerning a better understanding of signaling pathways inside CTCs, we also extracted a signaling subnetwork from the KEGG database. To determine the metastasis potentials of identified subnetworks, we carried out the SVM, neural network, and decision tree classifications on GSE7390, and the selected model was validated in GSE9195. We also developed a metastasis-free-survival-risk model to predict patients' risk, using the VIF-stepwise feature selection and cox-PH model. Finally, an article review was implemented to detect novel metastatic biomarkers in breast cancer. 


\section{$93 \quad 2 \mid$ Results}

\section{$94 \quad 2.1 \mid$ Pre-Processing of CTCs and DEA}

95 After pre-processing, 74 out of 77 cells were included in the downstream analyses. The

96 excluded cells revealed low quality. The differential expression analysis was implemented after

97 the normalization step (FDR <0.05) [15]. The adjusted p-values and logarithm of fold changes

98 were reported in Additional file 2: Table S1 and Additional file 3: Table S2. As it is shown in

99 Figure 1, the downregulation of immune subnetwork was detected in CTC clusters (light purple

100 color in the heatmap); and the genes of the EMT subnetworks implied upregulation (dark

101 purple color in the heatmap) in CTC clusters. Moreover, the single and cluster CTCs grouped

102 well in both subnetworks (Figure 1a-b). The immune-related subnetwork represented a stronger

103 expression difference between clustered and single cells. 
(a) Heatmap of EMT subnetwork

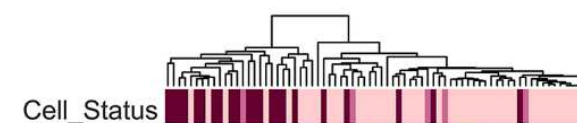

Cell_Status [II|| | | | || |

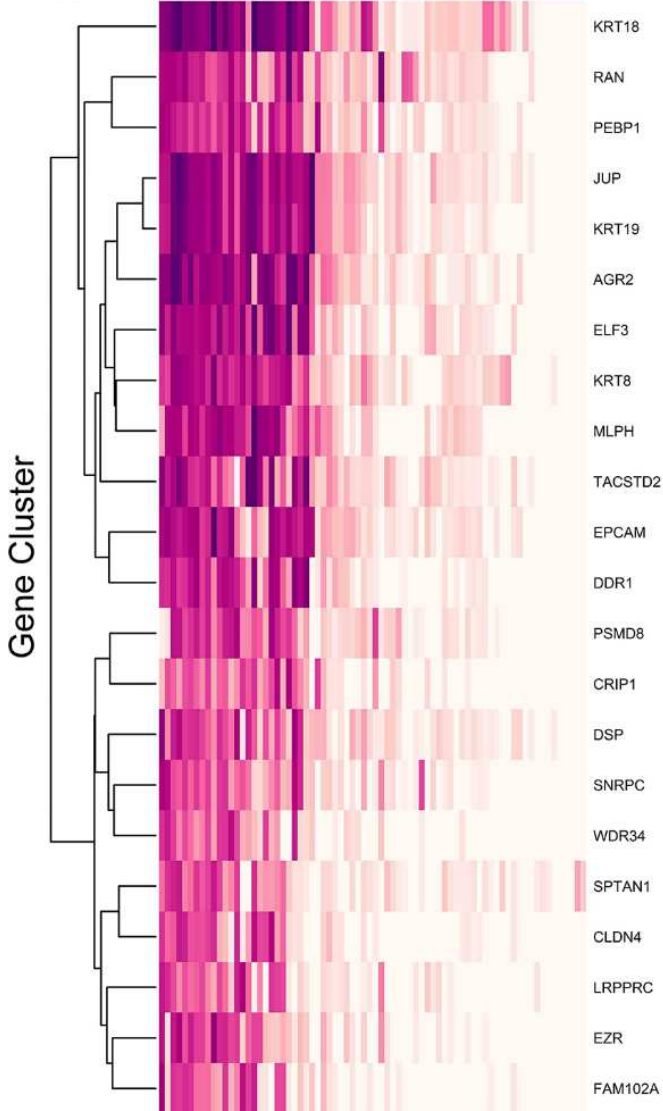

(b) Heatmap of immune subnetwork
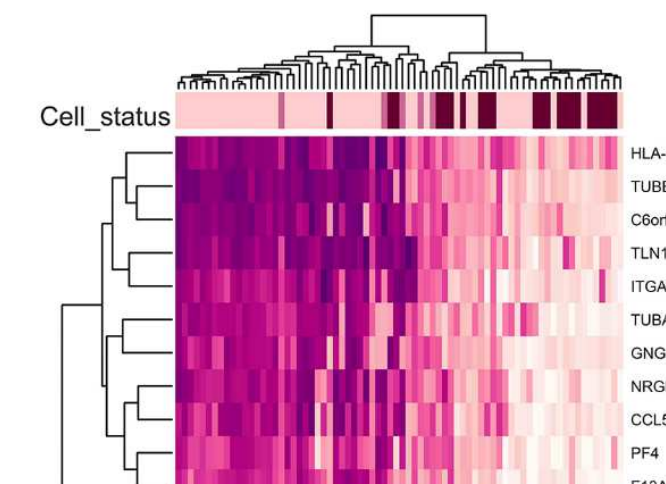

|
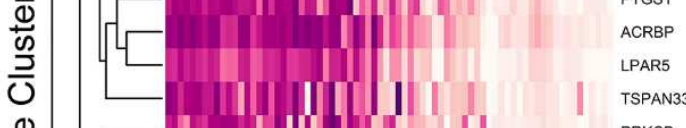

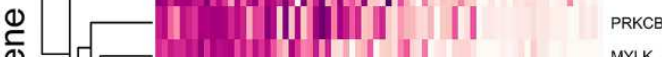

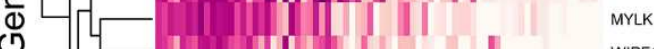

WIPF1

RGS18

FYB

SELP

CLEC1B

C2orf8

GFI1B

III $\operatorname{MAX}$

SLC2A3

DAB2

ICAM2

C17or72

PTCRA

P2RX

SDPR

Figure 1 CTC cluster vs. single CTCs gene expression change

(a) Gene expression changes between CTC clusters and single CTCs for EMT-related subnetwork 


\section{$109 \quad 2.2 \mid$ Metastasis Associated Subnetworks}

110 Metastasis-associated subnetworks were determined, using co-expression analysis and

111 hierarchical clustering [16]. We detected 16 subnetworks. The first principle component (in

112 PCA analysis) of subnetworks and the trait (cluster CTCs vs. single CTCs) association was

113 assessed by correlation analysis. The two top significant subnetworks, which had the highest

114 correlation with the trait, nominated for enrichment analysis

115 (|midnightblue correlation $\mid=0.57$, |turquoise correlation $\mid=0.51$ ) (Additional file 1: Figure

116 S1). The sizes of midnightblue and turquoise subnetworks were 35 and 22 genes, and they were

117 enriched for immune responses and EMT pathways, respectively (Figure 2). 
(a) Enrichment of EMT subnetwork

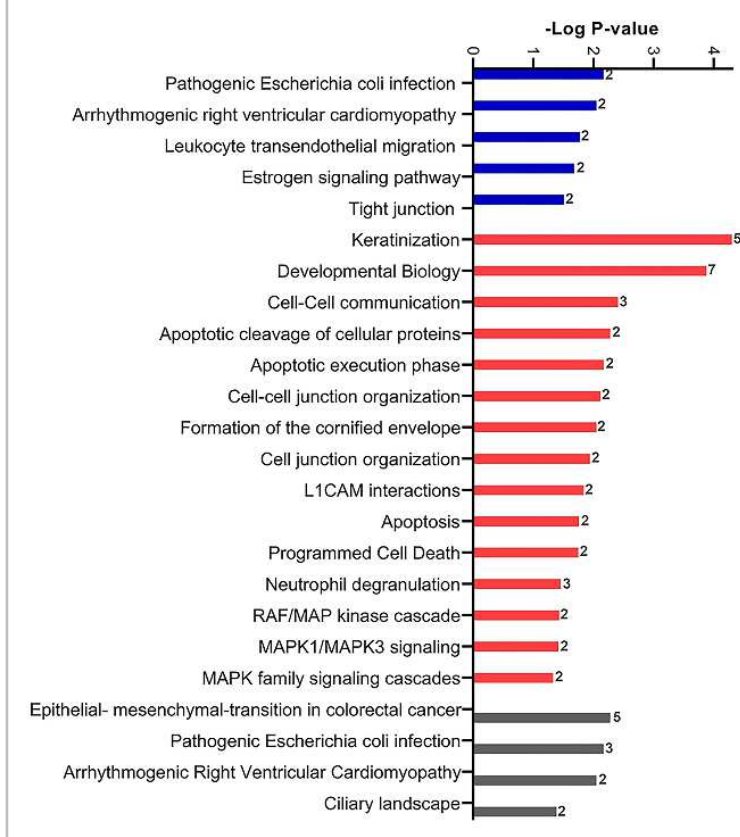

(b) Enrichment of immune subnetwork

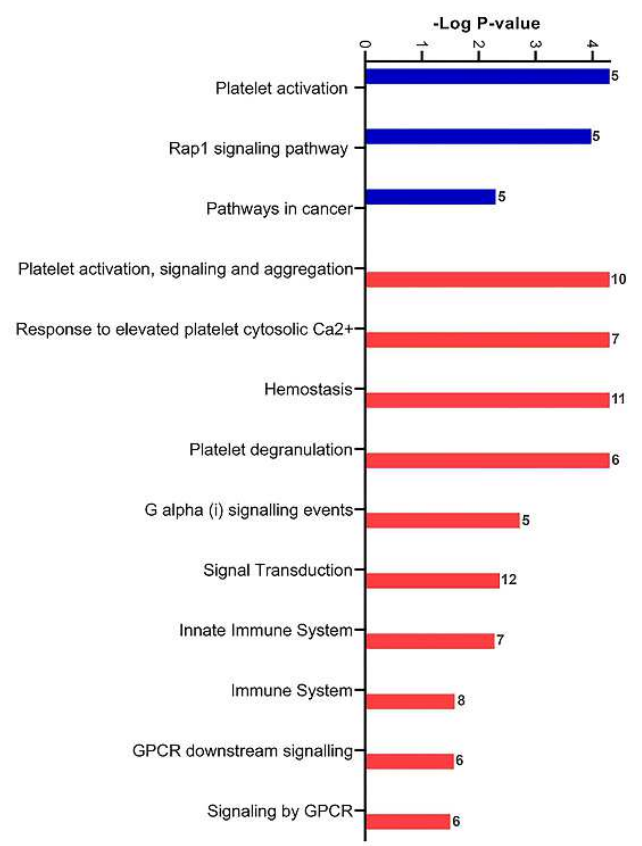

(c) Enrichment of signaling network

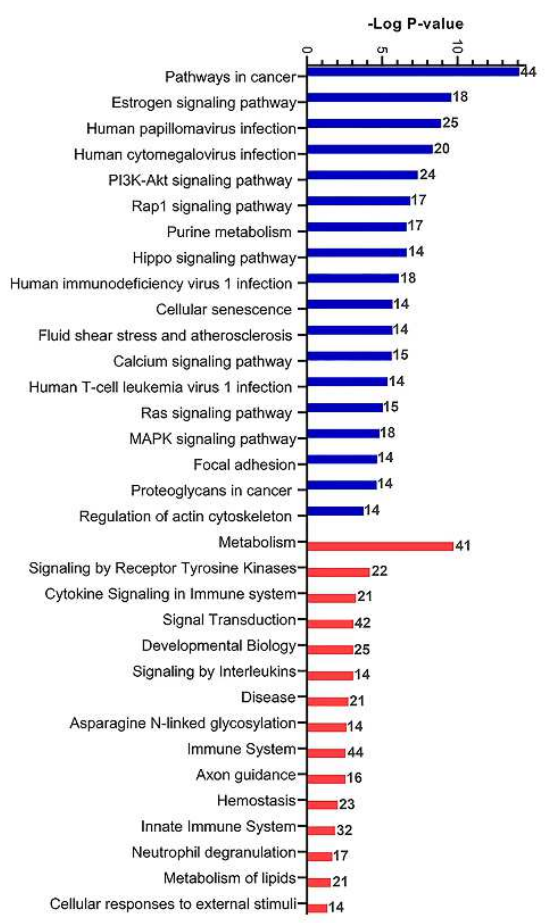

\section{Database indicator:}

| WIKIpathways database

I Reactome database

I KEGG database

119 Figure 2 Gene set enrichment analysis.

120 The numbers for each bar indicate the number of significant genes.

(a) Significant pathways of EMT subnetwork(size $=22)(\mathrm{q}$-value $<0.05)$

(b) Significant pathways of Immune subnetwork (

(c) Significant pathways of signaling network of CTCs (q-value $<0.05)$ 
125 To have a biological concept for subnetworks, we addressed the midnightblue and the turquoise

126 subnetworks, the Immune and EMT subnetworks, respectively, The EMT subnetwork included

127 cancer-related pathways such as 'cell-cell communication', 'tight junction', 'keratinization',

128 and 'estrogen signaling pathway'. The Immune subnetwork included pathways such as 'platelet

129 activation', 'immune system', and 'innate immune system'. After having reviewed the

130 literature, we detected the novel metastatic biomarkers in breast cancer. The immune-related

131 novel metastatic biomarkers in breast cancer were PTCRA, F13A1, LAT, GNG11, ICAM2,

132 NRGN, P2RX1, CLEC1B, BIN2, LPAR5, CCL5, SELP, RUFY1, C6orf25, TUBB1, GFI1B,

133 C2orf88, ACRBP, and C17orf72. Module membership and gene significance of Immune

134 subnetwork were reported in Additional file 2: Table S1.

135 The EMT related genes were LRPPRC, AGR2, CLDN4, CRIP1, DSP, ELF3, JUP, KRT8, 136 KRT18, KRT19, FAM102A, TACSTD2, EPCAM, PEBP1, PSMD8, RAN, SNRPC, SPTAN1,

137 EZR, DDR1, MLPH, and WDR34. In which, gene SNRPC, upregulated in CTC clusters, is a

138 metastatic novel biomarker in breast cancer. Module membership and gene significance for

139 EMT subnetwork were summarized in Additional file 3: Table S2.

140 The preservation of all subnetworks was assessed in the external dataset (GSE51827). The two

141 combined statistics $Z_{\text {summary }}$ and Median $_{\text {rank }}$ were calculated to assess subnetworks

142 preservation in the second dataset (Immune subnetwork: $Z_{\text {summary }}=14$, Median $_{\text {rank }}=9$

143 and EMT subnetwork: $Z_{\text {summary }}=31$, Median $_{\text {rank }}=6$ ). The $Z_{\text {summary values for both }}$

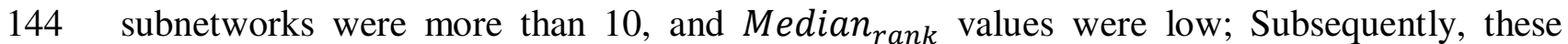
145 statistics indicated the Immune and EMT subnetwork preservations in the second dataset. The 146 statistics for all subnetworks reported in Additional file 4: Table S3). 


\section{$147 \quad 2.3 \mid$ Directed Network Reconstruction}

148 The signaling crosstalk between two selected subnetworks, Immune and EMT, was

149 investigated by mapping them to KEGG and extracting induced subnetwork. A directed

150 subnetwork of size 255 genes was extracted and illustrated in Figure 3. The network density

151 was 0.5 and it included 40 components, in which, PLCG1 showed the highest betweenness

152 value; and MYC, MYLK, and MRAS showed the highest closeness in the subnetwork.

153 We could detect 12 gene categories based on biological processes, including 'Hormonal

154 regulation', 'Immune responses', 'Ion metabolism', 'Nucleobase metabolism', 'Oxidative

155 responses', 'Protein localization', 'Protein topology response', 'STAT singling pathway',

156 'Vitamin metabolism', 'Cell differentiation', 'Circulation in blood regulation', and 'Energy

157 metabolism' (Figure 3). These categories were illustrated by colors on the network nodes, and

158 the genes with no category remained grey. The nodes with multiple colors indicated different

159 biological processes. The node size was illustrated by node degrees (Figure 3). PLCG1 and

160 ENTPD8 participated in 'Energy Metabolism' and 'Nucleobase Metabolism', were two hub

161 nodes in our detected directed network. The ClueGO results were reported in Additional file 5:

162 Table S4. 


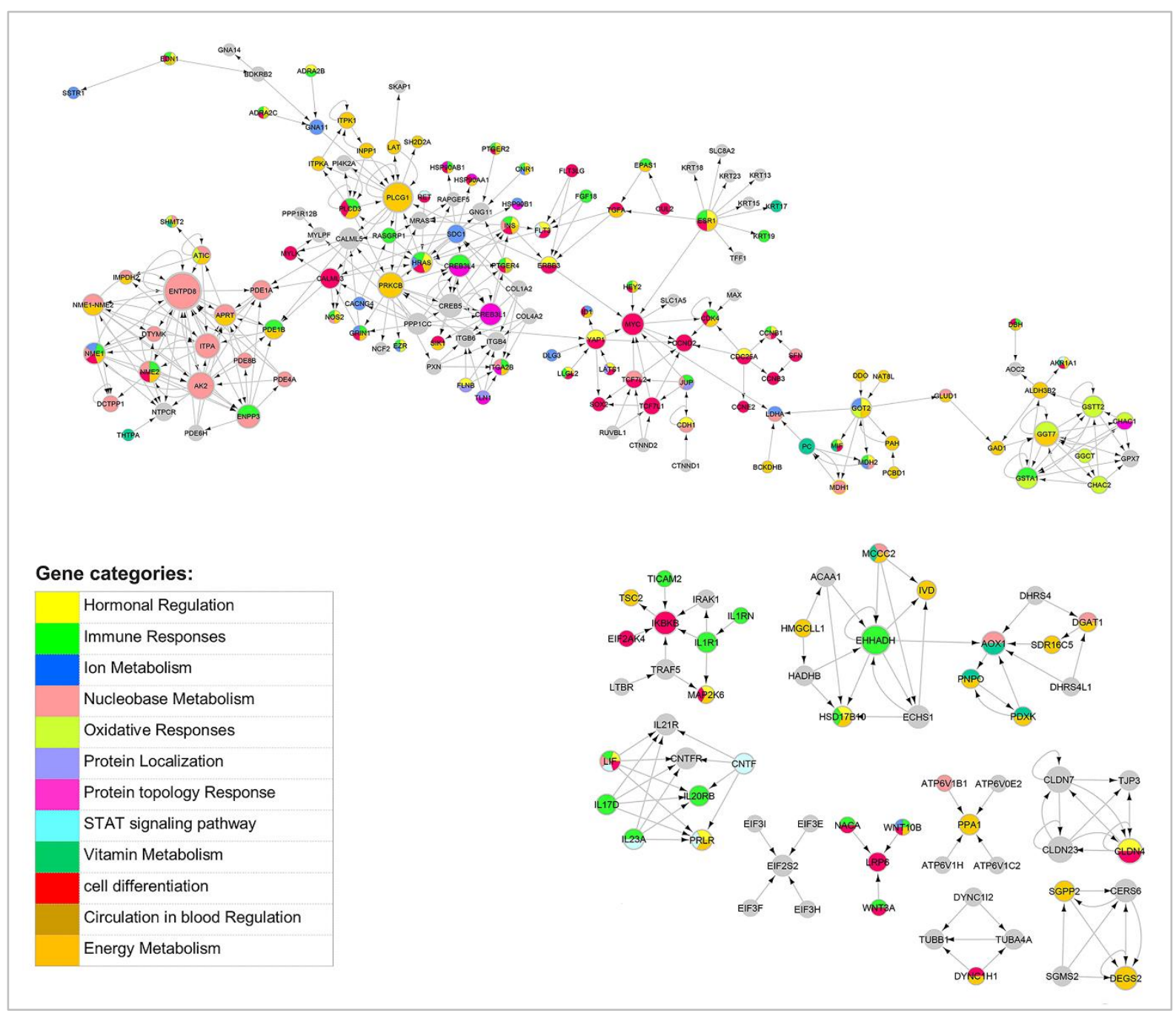

165 The node size indicates the node degree. The direction among genes is based on KEGG directions.

\section{4 | Distant Metastasis Classification Model}

168 The distant metastasis potential of two nominated subnetworks was assessed using SVM,

169 neural network, and decision tree classification methods in GSE7390. The accuracy,

170 sensitivity, and specificity scores of the SVM model for EMT subnetwork were 79\%, 78\%, and

$17121 \%$, respectively. The neural network accuracy, sensitivity, and specificity scores were $18 \%$,

$17218 \%$, and $80 \%$, respectively. Eventually, the decision tree accuracy, sensitivity, and specificity

173 scores were $60 \%, 60 \%$, and $30 \%$, respectively. These results refer to a full model (all genes in 
the subnetwork included). Comparing three models, the SVM model was the strongest method in classifying metastatic and non-metastatic patients, but the specificity score was too low.

176 The SVM accuracy, sensitivity, and specificity scores for immune-related subnetwork were $17778 \%, 78 \%$, and $78 \%$, respectively. The neural network accuracy, sensitivity, and specificity 178 scores were $85 \%, 85 \%$, and 14\%, respectively. Eventually, the decision tree accuracy, 179 sensitivity, and specificity scores were $71 \%, 71 \%$, and $36 \%$, respectively. These results refer 180 to a full model (all genes of subnetwork included). The specificity of the neural network and 181 decision tree methods was low compare to the SVM model. Due to the results, the SVM model 182 was the most powerful method in classifying metastatic and non-metastatic patients for the 183 immune-related subnetwork. The SVM model accuracy, sensitivity, and specificity for the 184 Immune subnetwork were superior to EMT subnetwork.

185 The feature selection algorithms, for the SVM model, were implemented for both subnetworks.

186 The WCC introduced 13 and 15 genes, and GA introduced 12, 17 genes for EMT- and immune187 related subnetworks, respectively. The WCC introduced HLA-E, MYLK, WIPF1, TLN1, 188 F13A1, NRGN, ICAM2, PTGS1, SELP, PF4, ITGA2B, GFI1B, TUBB1, PTCRA, RUFY1, 189 BIN2, and CLEC1B and the GA introduced CCL5, MYLK, WIPF1, TLN1, NRGN, GNG11, 190 PTGS1, SELP, ITGA2B, MAX, GFI1B, P2RX1, PTCRA, RUFY1, and BIN2 for the immune 191 subnetwork.

192 The SVM model, full model, for immune subnetwork validated in GSE9195. The accuracy, 193 sensitivity, and specificity were 0.868; surprisingly, the validation scores were superior to 194 GSE7390. The results confirmed that the immune-related genes detected in this study can 195 classify metastatic and non-metastatic samples more precisely compared to the neural network 196 and decision tree models, using two data sets. We implemented the classification methods to 197 assess the metastasis potential of two nominated CTC-related subnetworks. 


\section{5 | Distant Metastasis-Free-Survival and Overall Survival Analyses}

199 The association between gene expression and distant metastasis-free survival /overall survival

200 was implemented to detect metastatic potential genes in two selected subnetworks. Overall 201 survival and distant metastasis-free survival of JUP, KRT18, and KRT19 were significant (

202 Log-rank test p-value $<0.05$; the exact p-values were reported in Figures) (Figure 4a-f). These

203 three genes belonged to EMT subnetwork. The upregulation of JUP, KRT18, and KRT19 was

204 associated with more metastases; Therefore, the lower overall survival of patients (Figure 4a-

205 f). Moreover, JUP, KRT18, and KRT19 were upregulated in CTC clusters (Figure 1a). The

206 lower distant metastasis-free survival and lower overall survival curves confirmed the

207 importance of selected genes in metastasis. Therefore, they are important gene signatures in

208 CTCs.

209 We fitted a metastasis free-survival Cox-PH regression model for EMT and Immune

210 subnetworks to assess patients'metastasis risks through a predictive model. The Immune Cox-

211 PH model included RUFY1 and P2RX1 variables (Likelihood ratio test $\mathrm{p}$-value $=0.0295$ ); and

212 the EMT Cox-PH model included of RAN, PEBP1, KRT8, DSP, DDR1, and CLDN4 variables

213 (Likelihood ratio test $\mathrm{p}$-value $=0.0001016$ ). The coefficients of variables and $\mathrm{p}$-values were

214 reported in Additional file 6: Table S5 and Additional file 7: Table S6. All the significant genes

215 in the model had VIF $<2$ to avoid multicollinearity problems, using the VIF-feature selection

216 method (Additional file 8: Table S7 and Additional file 9: Table S8). The proportional hazard

217 assumptions for two model variables were assessed by the Schoenfeld residuals (Additional

218 File 10: Figure S2 and Additional File 11: S3). The predictive Cox-PH models for distant

219 metastasis-free survival for two subnetworks were illustrated in Figure $4 \mathrm{~g}, \mathrm{~h}$. The concordance

220 index, as a performance evaluation measure, for EMT and Immune predictive Cox-PH models

221 were 0.7 and 0.6 , respectively. Therefore, the EMT model is more powerful in discriminating 


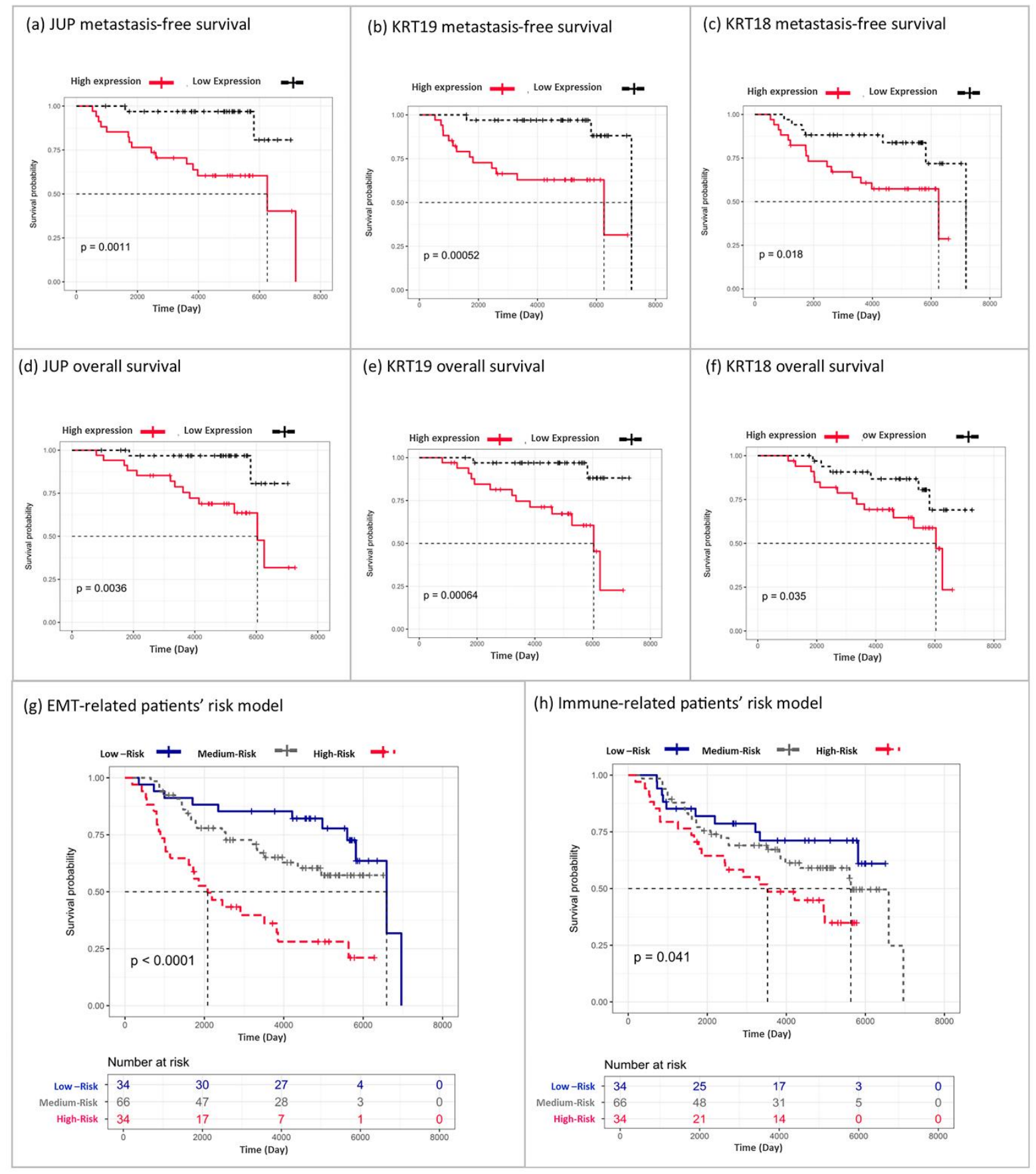

Figure 4 Metastasis free survival and overall survival.

p indicate p-value of Log-Rnk test in a,b,c,d,e,f,g, and h section.

(g) a predictive metastasis risk model for EMT subnetwork. High risk indicates upper-quartile of gene expression; low-risk indicates lower-quartile of gene expression.

(h) a predictive metastasis risk model for the immune subnetwork. High risk indicates upper-quartile of gene expression; low-risk indicates lower-quartile of gene expression. 


\section{3 | Discussion}

233 Whereas multiple studies on circulating tumor cells (CTCs) as single CTCs or metastatic

234 microemboli (CTC clusters) have been conducted, the molecular mechanisms of such rare cells

235 are insufficiently characterized. CTCs bear several undiscovered metastatic potentials to

236 overcome many restrictions, including extravasation of the primary tumor microenvironment,

237 survival in the bloodstream, and successfully colonize secondary organs; Therefore, a better

238 understanding of the biological mechanisms of different types of CTCs, single/cluster, is

239 essential.

240 This study was aimed to explore metastasis-related mechanisms within CTCs. We have

241 implemented the co-expression analysis to detect subnetworks discriminating single/cluster

242 CTCs (Figure 1). Two of subnetworks indicated a significant correlation to the trait

243 (single/cluster status of CTCs). Our detected subnetworks illustrated immune- and EMT-

244 related pathways (Figure 2a-b). Due to previous studies, the immune-associated mechanisms

245 and EMT pathways are of two major arms in breast cancer progression and metastasis, but

246 investigating them in CTCs is not thoroughly studied [11]. To prepare cancer cells for

247 intravasation in early stages, the keratin family, claudins, and cadherins must be downregulated

248 through the EMT process in primary tumors; still, due to the surviving urgency of CTCs in the

249 bloodstream and avoiding anoikis, a small number of tumor cells must be attached and break

250 off from the primary site [17, 18]. Therefore, the keratins, claudins, and cadherins should be

251 upregulated in CTC clusters to survive shear forces in blood circulation. The plakoglobin

252 (JUP), KRT8, KRT18, KRT19, CLDN4, and EPCAM, which are EMT-related biomarkers, are

253 such essential genes that their role in breast cancer metastasis demonstrated in several studies

$254[5,18,19]$. The KRT8, KRT18, KRT19 are a group of cytoskeleton genes within the cellular 
cytoplasm called keratins. Although they are extensively used as diagnostic tumor markers, several studies have demonstrated their involvement in cancer cell invasion and metastasis, as well as in treatment responsiveness $[19,20]$. Keratins are the intermediate filament-forming proteins of epithelial cells that organize the internal three-dimensional cellular structure; In fact, they act in cell shape maintenance for bearing tensions. [20]. Moreover, the plakoglobin, the upregulation of which in breast cancers CTC clusters in comparison to single cells was demonstrated in Aceto, Nicola, et al. study, is one of the cell junction genes that hold tumor cells together to leave primary tumor as CTC clusters [5]. In our study, the overexpression of JUP, KRT18, and KRT19, as well as the overall survival and metastasis-free survival were significant (Figure 4a-f). Therefore, they may play an essential role in the integrity of CTC clusters in the bloodstream shear forces. Meanwhile, EPCAM and cytokeratins have been reported as detection markers in the enrichment of CTCs [21]. Of note, few markers guide scientists to detect metastatic patients in the clinic therefore the rest of the genes detected in EMT subnetwork were not investigated in CTC studies; Consequently, they could be new targets in experimental studies for CTCs.

Several types of immune cells ambiguously reveal anti- and pro-tumor behaviors [22]. The immunosuppressive microenvironment of tumors protects the primary tumor cells.

272 Nevertheless, while tumor cells extravasate and enter circulation, they lose their tumor 273 protection; Therefore, they must adapt themselves to escape immune surveillance [1, 22]. The 274 interplay between immune cells and cytokeratins may contribute to evasion of CTCs from immune surveillance. The cytotoxic T lymphocytes (CTLs) were recruited by recognizing tumor antigens presented by major histocompatibility class I (MHCI) [23, 24]. The underexpression of MHCI in tumor cell surface guides them to hide from CTLs and thereby survive 278 in circulation. Moreover, the overexpression of cytokeratins such as KRT8, and together with 
heterodimeric partners KRT18 and KRT19 inhibit MHCI interactions with CTLs [22, 23]. All

280 these findings, overexpression of KRT8, KRT18, KRT19, and under-expression of HLA-E, are

281 consistent with our results which highlight the CTC cluster potential to evade the immune

282 system; consequently, longer survival (Figure 1a-b).

283 Of note, several studies supported the association between EMT and immune cell escape of cancer cells $[25,26]$. Moreover, a plethora of genes and signals support stemness pathways such as Wnt, TGF- $\beta$, and NOTCH in CTCs [10]. Downregulation of DAB2, which is a putative tumor suppressor and involves in TGF- $\beta$ pathway and promotes EMT, was reported in breast cancer tumors $[27,28]$. Therefore, the under-expression of DAB2 in CTC clusters might be related to the stemness phenotype which helps CTC clusters to escape the immune system. In our study, DAB2 the expression of which was downregulated in CTC clusters, the logarithm of fold change $=-5.7$, was detected in our immune subnetwork (Figure 1b). Therefore, our findings may indicate the survival potential of CTC clusters in circulation, which were consistent with previous studies on cancer biology. Whereas the CTC clusters have higher metastatic potential due to less frequency in metastatic patients, but the single cells contribute metastasis either. Hereof, several studies such as Szczerba, Barbara Maria, et al. indicated more single-cell, about $88.0 \%$, detected in metastatic patients [29]. Therefore, either single CTCs or CTC clusters have metastasis potential with different molecular mechanisms.

297 Not only the immune system and EMT but also the intermediate pathways are important in the progression of CTCs. The crosstalk between the signaling pathways of immune response and hormonal regulations, such as the fluctuation of menstrual cycles, were investigated by

300 Atashgaran, Vahid, et al. in breast cancer [30]. Furthermore, they demonstrated the dis301 regulation of hormonal factors affecting genome instability and the decrease of immune surveillance in breast cancer [31]. We know EMT is a complex process through which tumor 
303 cells facilitate their dissemination and acquire stemness characteristics [14, 32]. Not only

304 signaling pathways of stemness but also in the stimulation of self-renewing pathways in tumor

305 cells are essential in embryogenesis $[14,33]$. Such several metastatic prone pathways and the

306 interplay among all of them activated in circulating tumor cells (Figure $2 \mathrm{c}$ and Figure 3). The

307 CTCs are tumor cells that reflect characteristics of primary tumors and likewise more additional

308 metastatic propensity to survive in blood-stream and extravasate secondary site. These pro-

309 metastatic cells need to recruit different signaling pathways, the interrelationship of which

310 leads to creating multi-role cells that reflect great metastatic and survival potentials. As a result,

311 characterizing multiple aspects of CTC, involved in cancer progression is essential; Moreover,

312 useful in finding novel biomarkers or patients' treatment strategies.

\section{$3134 \mid$ Conclusions}

314 In summary, although CTCs, which are cancer-related biomarkers, are applied in the clinic, the

315 molecular mechanisms were not investigated well. The unknown crosstalks among multiple

316 pathways including EMT and immune responses improve the survival of CTCs in the patients'

317 blood. Therefore, they may contribute to therapeutic resistance and metastasis. Computational

318 investigations on CTCs suggest novel metastatic biomarkers which could be new targets for

319 experimental studies or therapeutic aims.

\section{$320 \quad 5 \mid$ Methods}

\section{$321 \quad 5.1 \mid$ Data sets and Metadata Information:}

322 The single-cell RNA-seq data related to advanced ER+ breast cancer patients were

323 downloaded from the NCBI data repository (GSE86978). The data consist of 77 cells which

32447 of them were CTC clusters, 22 cells were single CTCs, and the rest of the cells were not 
categorized. The GSE51827, which consists of 29 cells (15 single CTCs and 14 CTC clusters), was used for subnetwork preservation analysis. The gene expression of GSE7390, which consists of 198 untreated breast cancer patients, was used for assessment of metastasis potential of subnetworks. The GSE9195, which consists of 77 breast cancer patients, was used for validation of the classification model, overall survival, and metastasis-free survival analyses.

\section{2 | Pre-Processing, Normalization, and Differential Analysis}

331 To have more precise downstream analysis and remove non-biological variations, we implemented several pre-process steps on genes and also cells. At the first step, we filtered out low abundance genes, then the small count cells omitted subsequently. In the last step, the expression data were normalized to reduce technical effects, using the scatter package in $\mathrm{R}$ [34]. The differential expression analysis (DEA), which compares clustered cells' expression to the single cells' expression, was implemented using the limma package in $\mathrm{R}(\mathrm{FDR}<0.05)$

$337 \quad[15]$.

\section{3 | Co-Expression Network (CEN) Reconstruction and Subnetwork Extraction}

The co-expression network reconstructed using a weighted correlation network analysis (WGCNA) method [16]. The pairwise relation among genes was estimated using the Pearson correlation among genes. Concerning to have more connected subnetworks, we carried out the topological overlap matrix (TOM) and connectivity gene filtering (connectivity values less 0.1 were omitted) [16]. Higher connectivity values indicate more considerable co-expressed subnetworks $[16,35]$. Eventually, we used hierarchical clustering to extract subnetworks. The trait used in this study was the cluster and single status of the cells captured in blood. The subnetworks, which have strong correlations between their first principle component and the biological trait, were selected as trait related (metastasis) subnetworks. The gene significance and module membership were used to filter essential genes in selected subnetworks. The gene 
significance is the correlation between gene expression and the metastasis trait. The module membership is the correlation between gene expression and module representative (first

351 principle component in the principal component analysis (PCA)). The preservation of subnetworks were assessed in the external dataset (GSE51827)

\section{4 | Signaling Network Reconstruction}

354 We downloaded all homo sapiens pathways from the Kyoto Encyclopedia of Genes and

355 Genomes (KEGG) database resource and merged them [36]. Furthermore, the KEGG ids were annotated to gene symbols. At the last step, to have casual relations among genes, we extracted a directed induced subnetwork from the KEGG database, using two detected significant subnetworks (|correlation |>0.5 was considered significant) [37]. The genes were categorized based on biological process (BP of gene ontology) terms, using the ClueGO plug-in in

360 Cytoscape $[38,39]$. The network visualization was implemented by the Cytoscape and the 361 Gephi software [39, 40].

\section{$362 \quad 5.5$ | Gene Set Enrichment Analysis and Subnetwork Preservation Analysis}

363 The significant trait-related, metastatic, subnetworks (|correlation $\mid>0.5$ ) were enriched, using

364 ConsensusPathDB webserver (q-value<0.05) [41]. The GSE51827, which has CTC gene 365 expression, downloaded from NCBI to implement preservation analysis of subnetworks in the 366 external dataset in $\mathrm{R}[16,35,42]$. The scatter package, which is suitable for single-cell RNA367 seq data, was used to preprocess, normalized, and merged expression data [34].

368 The combined statistics for preservation assessment, which includes $Z_{\text {summary }}$ and $369 M_{\text {Median }}$ rank, were used to check the reproducibility of subnetworks [35]. The $Z_{\text {summary }}$, 370 which shows the interaction pattern among genes in subnetworks, evaluate connectivity and

371 density in the external dataset. $Z_{\text {summary }}<2$ indicates not preserved subnetworks. If $2<$ $372 Z_{\text {summary }}<10$, the subnetwork is semi preserved, and if $Z_{\text {summary }}>10$, the subnetwork is 
373 preserved. Moreover, a higher Median $_{\text {rank }}$ indicates more preservation of subnetworks in the

374 external dataset [35]. The $Z_{\text {summary }}$ and Median rank $_{\text {were }}$ assessed for our subnetworks.

375

376

377

378

379

380

381

382

383

384

385

386

387

388

389

390

391

392

393

394

395

396

\subsection{Assessment of Distant Metastasis Potential of Subnetworks}

To evaluate the importance of selected subnetworks and the metastasis potential of genes, we implemented the classification algorithms on two individual datasets (GSE7390 and GSE9195). The GSE7390 (Affymetrix platform, HG-U133A) downloaded using the GEOquerry package in R [42]. The ER+ patients (134 patients out of 198 ones) filtered, and the expression data normalized using the RMA method [43]. In this section, we learned three classifiers, including support vector machine (SVM), artificial neural network (ANN), and decision tree on metastatic and non-metastatic patients [44]. The classification algorithms were run with and without feature selection algorithms, including the genetic algorithm (GA) and the world competitive contest (WCC) algorithm. The SVM was implemented with 5-fold crossvalidation, linear kernel, and 80 percent of cells as the training set. Finally, the accuracy, precision, and specificity were checked to select a better classifier for metastasis prediction; Furthermore, to identify the most metastatic-related subnetwork. To assess the reproducibility of our results, the selected model was validated in another dataset (GSE9195).

\section{7 | Distant Metastasis-Free Survival Analysis}

The Kaplan-Meier curve, distant metastasis-free survival, and overall survival analyses were implemented using GSE7390 in R [45]. The patients were stratified due to quartiles. The expression values lower than the first quartile were labeled low expression, and expression values higher than the third quartile were labeled high expression. The stepwise Cox proportional hazard ratio (Cox-PH) was implemented for selected subnetworks [45]. The concordance index was calculated to evaluate model performance. The Variance Inflation Factor (VIF) lower than two was used as the variable selection criteria. The first and third 
397 quartiles of the predicted hazard ratio were used for stratifying patients into three groups, 398 including low-risk, medium-risk, and high-risk groups.

$399 \quad$ List of abbreviations

400 CTC: circulating tumor cell

401 EMT: epithelial-mesenchymal-transition

402 SVM: Support vector machine

403 PTCRA: Pre T Cell Antigen Receptor Alpha

404 F13A1: Coagulation Factor XIII A Chain

405 LAT: Linker For Activation Of T Cells

406 ICAM2: Intercellular Adhesion Molecule 2

407 OS: overall survival

408 PCA: principal component analysis

409 WCC: world competitive contest

410 GA: genetic algorithm

411 ANN: artificial neural network

412 Cox-PH: Cox proportional hazard ratio

413 DEG: differentially expressed gene

414 VIF: Variance Inflation Factor

415 FDR: false discovery rate

\section{Declarations}

417 Ethics approval and consent to participate

418 Not applicable. 
419 Consent for publication

420 Not applicable.

421 Availability of data and materials

422 The public data were used in this article including GSE51827, GSE9195, GSE7390, and

423 GSE86978.

424 Competing interests

425 The authors declare that they have no competing interests.

\section{Funding}

427 Not applicable.

428 Authors' contributions

429 Conceptualization, Khoshbakht and Azimzadeh; Methodology, Khoshbakht and Azimzadeh;

430 Validation: Khoshbakht; Formal Analysis, Khoshbakht; writing-Original draft preparation,

431 Khoshbakht; writing, Review, and editing, Khoshbakht, Azimzadeh and Masudi-Nejad;

432 Visualization, Khoshbakht; Supervision, Masudi-Nejad and Azimzadeh. All authors have read

433 and agreed to the published version of the manuscript.

434 Acknowledgments

435 Not applicable.

436

437 


\section{References}

439 1. Leone, K., C. Poggiana, and R.J.D. Zamarchi, The interplay between circulating

$440 \quad$ tumor cells and the immune system: from immune escape to cancer immunotherapy.

441

442

443

444

445

446

447

448

449

450

451

452

453

454

455

456

457

458

459

460 2018. 8(3): p. 59.

2. Weigelt, B., J.L. Peterse, and L.J.J.N.r.c. Van't Veer, Breast cancer metastasis: markers and models. 2005. 5(8): p. 591-602.

3. Klein, C.A.J.N.R.C., Parallel progression of primary tumours and metastases. 2009. 9(4): p. 302-312.

4. Ghajar, C.M. and M.J.J.N. Bissell, Metastasis: pathways of parallel progression. 2016. 540(7634): p. 528-529.

5. Aceto, N., et al., Circulating tumor cell clusters are oligoclonal precursors of breast cancer metastasis. 2014. 158(5): p. 1110-1122.

6. $\quad$ Lang, J.E., et al., RNA-Seq of circulating tumor cells in stage II-III breast cancer. 2018. 25(8): p. 2261-2270.

7. Barneh, F., et al., Valproic acid inhibits the protective effects of stromal cells against chemotherapy in breast cancer: Insights from proteomics and systems biology. Journal of cellular biochemistry, 2018. 119(11): p. 9270-9283.

8. Yang, C., et al., Circulating tumor cells in precision oncology: clinical applications in liquid biopsy and 3D organoid model. Cancer Cell International, 2019. 19(1): p. 341.

9. Dasgupta, A., A.R. Lim, and C.M.J.M.o. Ghajar, Circulating and disseminated tumor cells: harbingers or initiators of metastasis? 2017. 11(1): p. 40-61.

10. Massagué, J. and A.C.J.N. Obenauf, Metastatic colonization by circulating tumour cells. 2016. 529(7586): p. 298-306.

461

462

463

464

465

466

467

468

469

470

471

472

473

474

475

476

477

478

479

480

481

482

483

11. Santisteban, M., et al., Immune-induced epithelial to mesenchymal transition in vivo generates breast cancer stem cells. 2009. 69(7): p. 2887-2895.

12. Pastushenko, I. and C.J.T.i.c.b. Blanpain, EMT transition states during tumor progression and metastasis. 2019. 29(3): p. 212-226.

13. Kudo-Saito, C., et al., Cancer metastasis is accelerated through immunosuppression during Snail-induced EMT of cancer cells. 2009. 15(3): p. 195-206.

14. Barneh, F., et al., Integrated use of bioinformatic resources reveals that co-targeting of histone deacetylases, IKBK and SRC inhibits epithelial-mesenchymal transition in cancer. Briefings in bioinformatics, 2019. 20(2): p. 717-731.

15. Smyth, G.K., Limma: linear models for microarray data, in Bioinformatics and computational biology solutions using $R$ and Bioconductor. 2005, Springer. p. 397420.

16. Langfelder, P. and S.J.B.b. Horvath, WGCNA: an R package for weighted correlation network analysis. 2008. 9(1): p. 559.

17. Fabisiewicz, A. and E.J.M.O. Grzybowska, CTC clusters in cancer progression and metastasis. 2017. 34(1): p. 12.

18. Joosse, S.A., et al., Changes in keratin expression during metastatic progression of breast cancer: impact on the detection of circulating tumor cells. 2012. 18(4): p. 9931003.

19. Tökés, A.-M., et al., Claudin-1,-3 and-4 proteins and mRNA expression in benign and malignant breast lesions: a research study. 2005. 7(2): p. R296.

20. Karantza, V.J.O., Keratins in health and cancer: more than mere epithelial cell markers. 2011. 30(2): p. 127-138. 
21. Deng, G., et al., Enrichment with anti-cytokeratin alone or combined with antiEpCAM antibodies significantly increases the sensitivity for circulating tumor cell detection in metastatic breast cancer patients. 2008. 10(4): p. R69.

22. Mohme, M., S. Riethdorf, and K.J.N.r.C.o. Pantel, Circulating and disseminated tumour cells-mechanisms of immune surveillance and escape. 2017. 14(3): p. 155.

23. Wu, M.-S., et al., Cytokeratin 8-MHC class I interactions: a potential novel immune escape phenotype by a lymph node metastatic carcinoma cell line. 2013. 441(3): p. 618-623.

24. Joosten, S.A., L.C. Sullivan, and T.H.J.J.o.i.r. Ottenhoff, Characteristics of HLA-E restricted T-cell responses and their role in infectious diseases. 2016. 2016.

25. Jia, D., et al., Quantifying cancer epithelial-mesenchymal plasticity and its association with stemness and immune response. 2019. 8(5): p. 725.

26. Terry, S., et al., New insights into the role of EMT in tumor immune escape. 2017. 11(7): p. 824-846.

27. Bagadi, S.A.R., et al., Frequent loss of Dab2 protein and infrequent promoter hypermethylation in breast cancer. 2007. 104(3): p. 277-286.

28. Martin, J., B.-S. Herbert, and B.J.B.j.o.c. Hocevar, Disabled-2 downregulation promotes epithelial-to-mesenchymal transition. 2010. 103(11): p. 1716-1723.

29. Szczerba, B.M., et al., Neutrophils escort circulating tumour cells to enable cell cycle progression. Nature, 2019. 566(7745): p. 553-557.

30. Atashgaran, V., et al., Dissecting the biology of menstrual cycle-associated breast cancer risk. 2016. 6: p. 267.

31. Atashgaran, V., et al., Dissecting the biology of menstrual cycle-associated breast cancer risk. Frontiers in oncology, 2016. 6: p. 267.

32. Takebe, N., R.Q. Warren, and S.P.J.B.c.r. Ivy, Breast cancer growth and metastasis: interplay between cancer stem cells, embryonic signaling pathways and epithelial-tomesenchymal transition. 2011. 13(3): p. 211.

33. Takebe, N., R.Q. Warren, and S.P. Ivy, Breast cancer growth and metastasis: interplay between cancer stem cells, embryonic signaling pathways and epithelial-tomesenchymal transition. Breast cancer research, 2011. 13(3): p. 211.

34. McCarthy, D.J., et al., Scater: pre-processing, quality control, normalization and visualization of single-cell RNA-seq data in R. 2017. 33(8): p. 1179-1186.

35. Langfelder, P., et al., Is my network module preserved and reproducible? 2011. 7(1).

36. Zhang, J.D. and S.J.B. Wiemann, KEGGgraph: a graph approach to KEGG PATHWAY in R and bioconductor. 2009. 25(11): p. 1470-1471.

37. Piran, M., et al., Can we assume the gene expression profile as a proxy for signaling network activity? 2020. 10(6): p. 850.

38. Bindea, G., et al., ClueGO: a Cytoscape plug-in to decipher functionally grouped gene ontology and pathway annotation networks. 2009. 25(8): p. 1091-1093.

39. Shannon, P., et al., Cytoscape: a software environment for integrated models of biomolecular interaction networks. 2003. 13(11): p. 2498-2504.

40. Bastian, M., S. Heymann, and M. Jacomy. Gephi: an open source software for exploring and manipulating networks. in Third international AAAI conference on weblogs and social media. 2009.

41. Kamburov, A., et al., ConsensusPathDB: toward a more complete picture of cell biology. 2011. 39(suppl_1): p. D712-D717.

42. Davis, S. and P.S.J.B. Meltzer, GEOquery: a bridge between the Gene Expression Omnibus (GEO) and BioConductor. 2007. 23(14): p. 1846-1847. 
532

533

534

535

536

537

538

539

540

541

$542 \quad(\mid$ correlation $\mid>0.5)$

543 Additional file 2:

544 Table S1. midnightblue (immune) subnetwork.

545 The list of genes, module membership, the logarithm of fold change, differential analysis

546 adj-p.value, and gene-significance statistics.

547 Additional file 3:

548 Table S2. turuise (EMT) subnetwork.

549 The list of genes, module membership, the logarithm of fold change, differential analysis

550 adj-p.value, and gene-significance statistics.

$551 \quad$ Additional file 4:

552 Table S3. The preservation statistics.

553 This table includes $Z_{\text {summary }}$ and Median rank $_{\text {statistics. }}$

554 Additional file 5:

555 Table S4. Biological pathways in the directed network.

556 We categorized genes of the detected directed network, using ClueGO plugin in Cytoscape.

557 The Biological pathways and p-values were reported in Table S4. 
558 Additional file 6:

559 Table S5. the EMT subnetwork cox-PH results.

560 The cox-PH analysis was implemented for the EMT subnetwork genes. The selected genes,

561 coefficients, and p-values were reported in Table S5.

562 Additional file 7:

563 Table S6. the Immune subnetwork cox-PH results

564 The cox-PH analysis was implemented for the immune subnetwork genes. The selected genes,

565 coefficients, and p-values were reported in Table S6.

566 Additional file 8:

567 Table S7. EMT VIF values.

568 To investigate multicollinearity in the cox-PH model, we calculated the Variance Inflation

569 Factor (VIF). The VIF $<10$ indicates no multicollinearity. The VIF of immune genes was

$570 \quad$ reported in Table S7.

$571 \quad$ Additional file 9:

572 Table S8. immune VIF values.

573 To investigate multicollinearity in the cox-PH model, we calculated the Variance Inflation

574 Factor (VIF). The VIF < 10 indicates no multicollinearity. The VIF of immune genes was

575 reported in Table S8.

576 Additional file 10:

577 Figure S1. The Schoenfeld residuals for EMT genes.

578 The proportional hazard ratio investigated, using the Schoenfeld residuals. The residuals (red 579 dots) must be between the curves.

580 Additional file 11:

581 Figure S2. The Schoenfeld residuals for immune genes. 
582 The proportional hazard ratio investigated, using the Schoenfeld residuals. The residuals (red 583 dots) must be between the curves.

584

585

586

587

588

589 
Figures

(a) Heatmap of EMT subnetwork

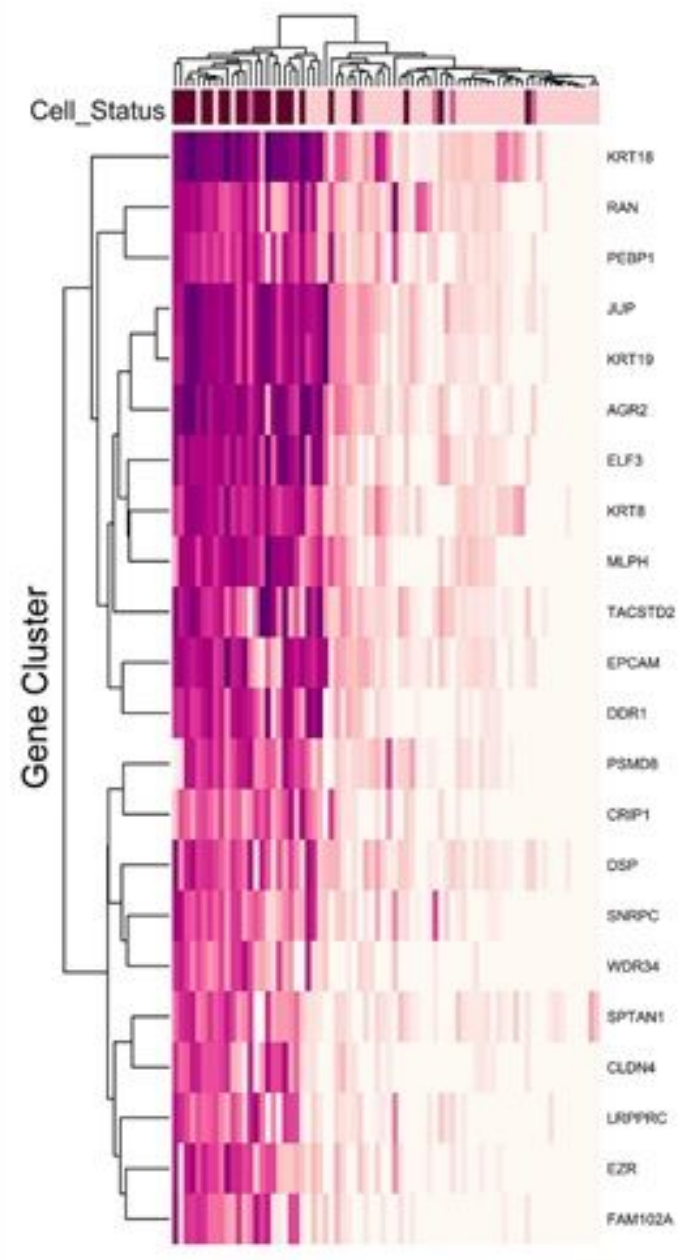

(b) Heatmap of immune subnetwork

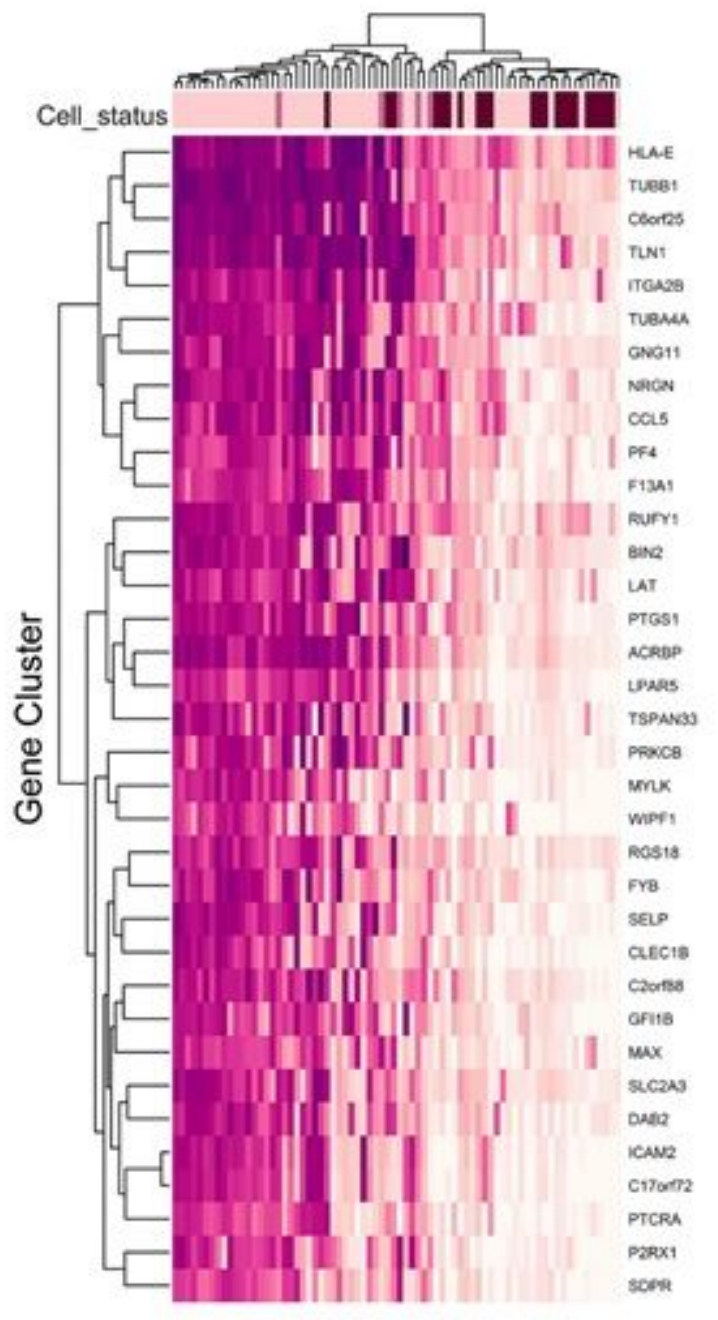

Expression Level
-20
15
10
5
0

Cell_status

cluster cells

Not available

single cell

\section{Figure 1}

CTC cluster vs. single CTCs gene expression change (a) Gene expression changes between CTC clusters and single CTCs for EMT-related subnetwork (b) Gene expression changes between CTC clusters and single CTCs for Immune-related subnetwork 
(a) Enrichment of EMT subnetwork

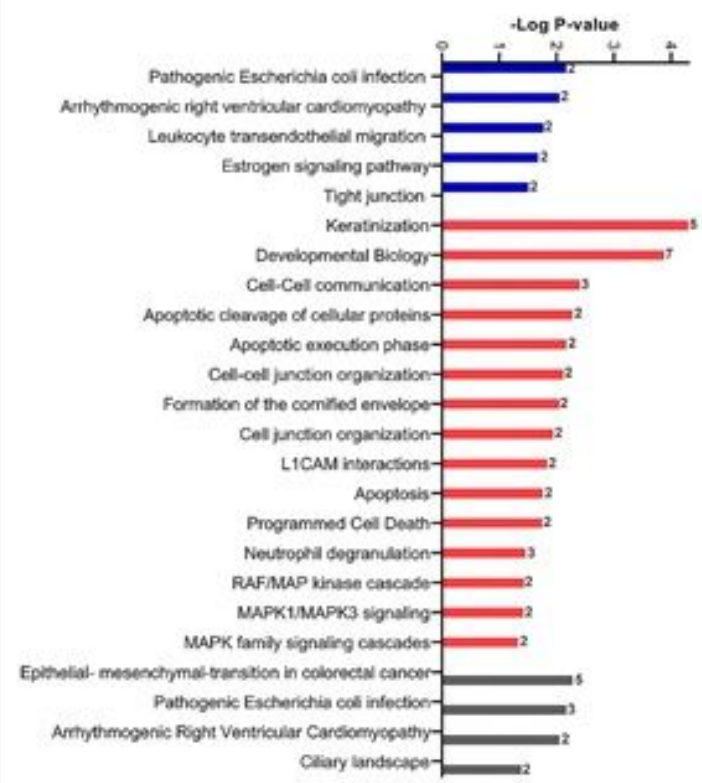

(b) Enrichment of immune subnetwork

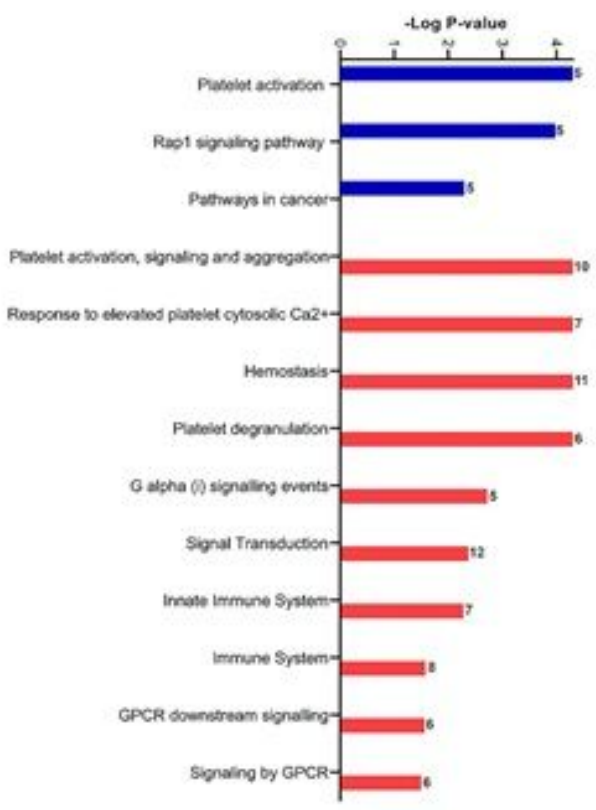

(c) Enrichment of signaling network

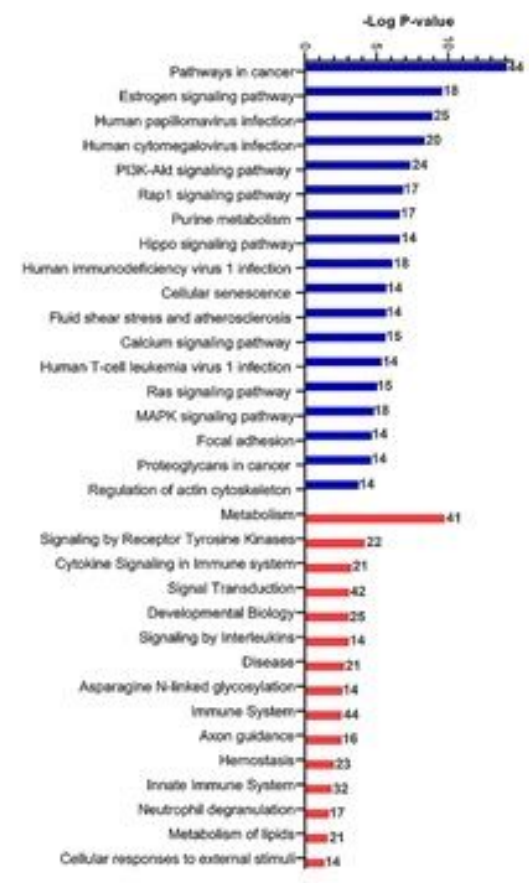

\section{Database indicator:}

| WIKIpathways database

I Reactome database

I KEGG database

\section{Figure 2}

Gene set enrichment analysis. The numbers for each bar indicate the number of significant genes. (a) Significant pathways of EMT subnetwork(size=22) (q-value<0.05) (b) Significant pathways of Immune subnetwork (size=35) (q-value<0.05) (c) Significant pathways of signaling network of CTCs (qvalue<0.05) 


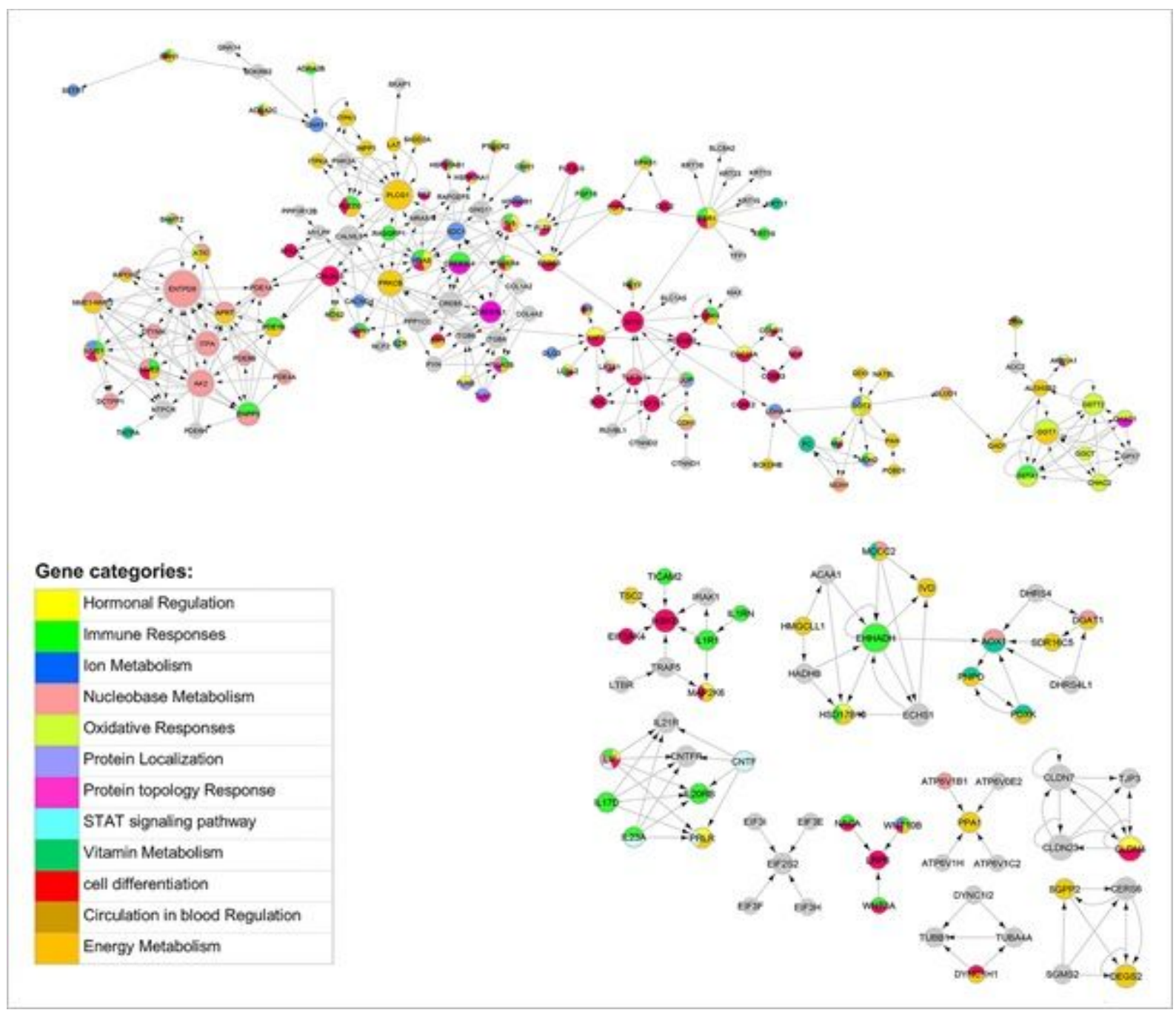

Figure 3

Signaling network of CTCs The node size indicates the node degree. The direction among genes is based on KEGG directions. 

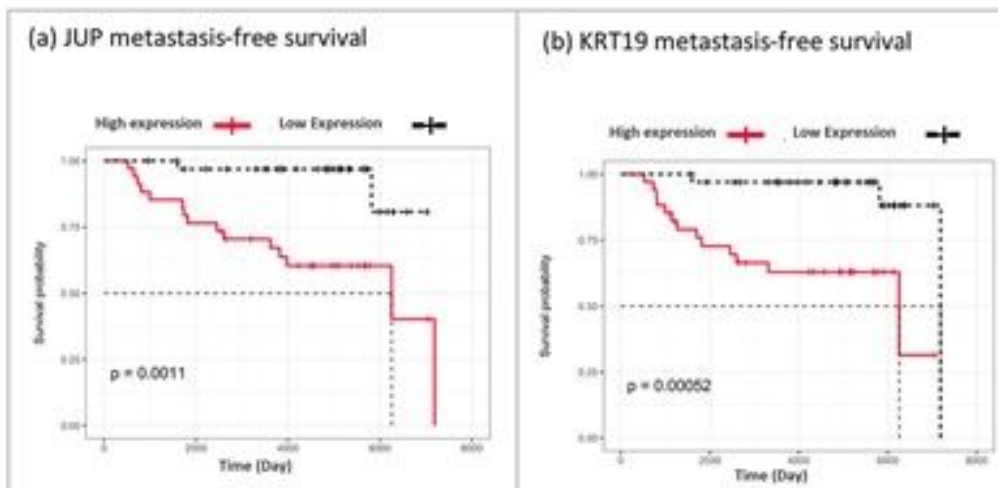

(c) KRT18 metastasis-free survival

(d) JUP overall survival

(e) KRT19 overall survival

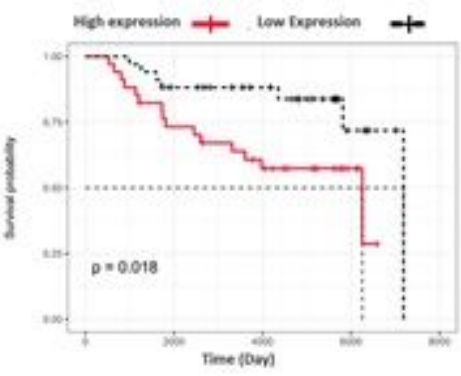

(f) KRT18 overall survival
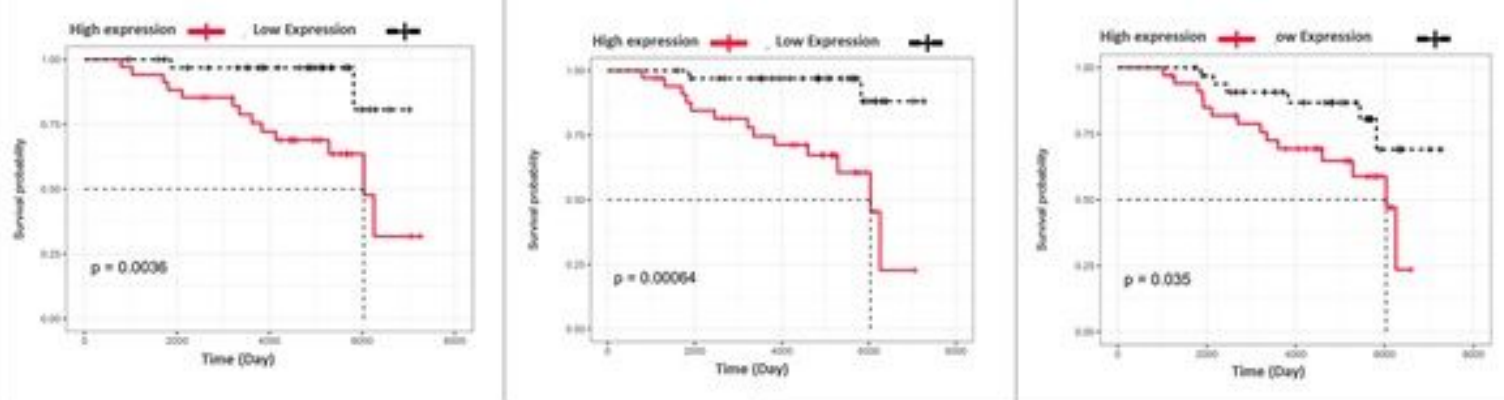

(g) EMT-related patients' risk model

(h) Immune-related patients' risk model
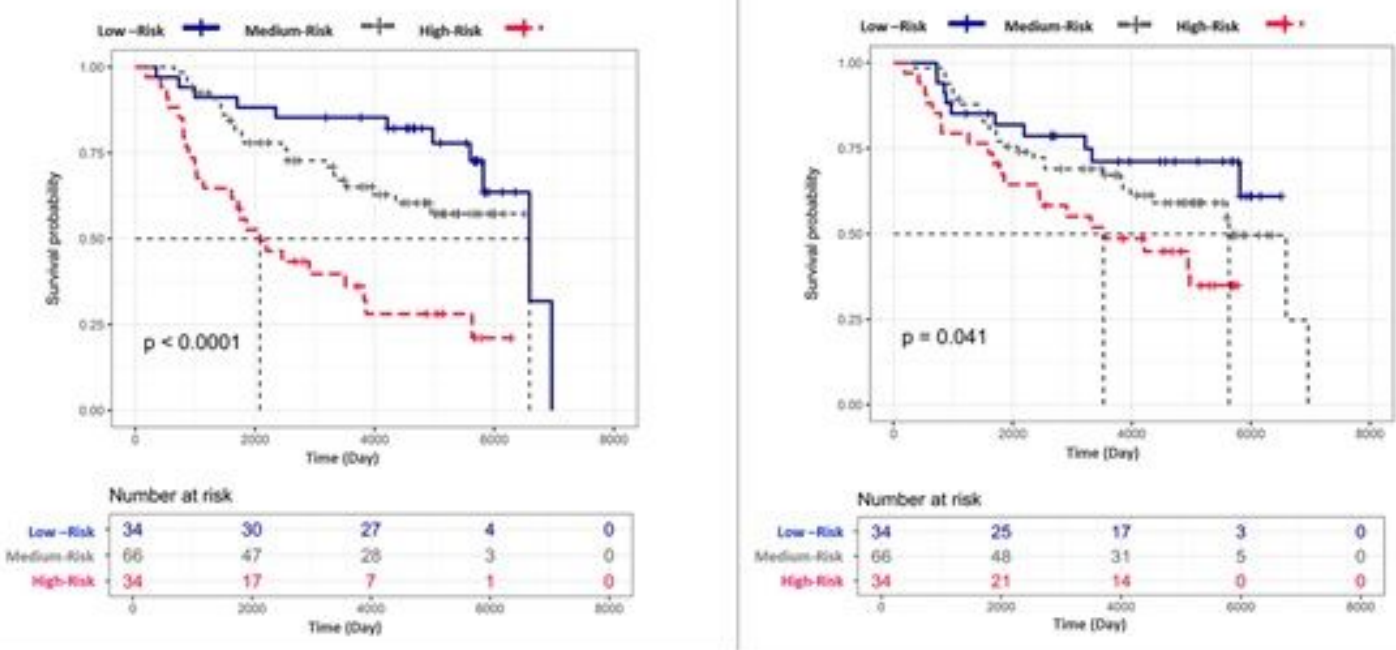

\section{Figure 4}

Metastasis free survival and overall survival. p indicate $p$-value of Log-Rnk test in a,b,c,d,e,f,g, and $h$ section. (g) a predictive metastasis risk model for EMT subnetwork. High risk indicates upper-quartile of gene expression; low-risk indicates lower-quartile of gene expression. (h) a predictive metastasis risk model for the immune subnetwork. High risk indicates upper-quartile of gene expression; low-risk indicates lower-quartile of gene expression.

\section{Supplementary Files}


This is a list of supplementary files associated with this preprint. Click to download.

- Supplementary.docx

- TableS1.xlsx

- Tables2.xlsx

- Tables3.xlsx

- TableS4.xIsx

- TableS5.xlsx

- Tables6.xIsx

- TableS7.xlsx

- Tables8.xlsx

- Figures1.tif

- Figures2.tif

- FigureS3.tiff 